qui, en toute justice, pouvait encore être attribuée à une légère erreur expérimentale. Un exemple frappant fut fourni par les échantillons de lait provenant d'un des régénérateurs. Cet appareil était construit de la façon suivante : les 6 premières conduites étaient de nickel pur ; elles étaient suivies de 8 conduites de cuivre, puis encore de 16 conduites de nickel. A l'entrée du régénérateur, l'analyse du lait indiqua une teneur en cuivre de 0,43 p. p. m. (1). Après le passage par les six conduites de nickel pur, la teneur en cuivre était tombée à 0,31 p. p. m. ; à la sortie des huit conduites de cuivre, la teneur en cuivre était devenue 0,76 p. p. m. ; à la sortie des dernières conduites de nickel, la teneur en cuivre du lait était revenue à 0,43 p. p. m.

Ces données ne sont que des exemples démontrant à quel degré la teneur en cuivre du lait varie au cours de son passage par une unité de pasteurisation. Nombre des conduites de nickel présentèrent, par endroits, une corrosion très prononcée, indiquant qu'il y avait eu perte de nickel, et, bien que la teneur en nickel du lait n'ait pas été déterminée, on peut présumer, sans crainte de se tromper, que lors du passage dans l'unité de pasteurisation, la teneur en nickel du lait était accrue à cause de l'absence d'un métal moins noble sur lequel le nickel aurait pu se précipiter.

Sans aucun doute, les données de détermination du cuivre du lait varieront de jour en jour, selon l'activité de l'usine et les températures de pasteurisation, mais nous sommes persuadés que toujours leurs rapports ressembleront à ceux observés dans ce cas particulier.

(A suivre.)

\title{
REVUE
}

\section{LA NOUVELLE LOI SUR LES PRODUITS LAITIERS}

\section{par Maxime TOUBEAU}

Inspecteur général, chef de service au Ministère de l'Agriculture.

En adoptant, sans modifications, le projet de loi voté en 1932 par la Chambre pour la protection des produits laitiers, le Sénat a marqué sa volonté de défendre, même au prix de nouvelles dérogations au principe de la liberté du commerce, notre agriculture contre certaines entreprises industrielles jugées dangereuses pour elle (2).

Le fait est assez important pour qu'on s'y arrête et qu'on l'étudie, dans ses origines et dans ses conséquences.

C'est un texte déposé sur le bureau de la Chambre, le 11 juillet

(1) p. p. m. = partie(s) par million.

(2) Loi du 29 juin 1934. Le Lait, n० 139, p. 1027. 
1930, par M. Fernand David, alors Ministre de l'Agriculture, qui a donné naissance à la loi nouvelle, promulguée en date du 29 juin 1934 et publiée au Journal Officiel du 1er juillet suivant.

M. Fernand DAvid avait eu son attention attirée sur la concurrence qui était faite à nos producteurs de lait par ceux qui substituaient, en tout ou en partie, des graisses animales ou végétales à la matière grasse du lait, dans la fabrication de certains produits tels que les crèmes et les fromages.

Au regard de la législation antérieure, et notamment de la loi du ler août 1905 sur la répression des fraudes, cette fabrication n'était pas répréhensible, dès l'instant:

$1^{\circ}$ que les produits fabriqués ne contenaient aucune substance nuisible à la santé du consommateur ;

$2^{\circ}$ que ees produits étaient mis en vente sous une dénomination appropriée, c'est-à-dire ne contrevenant à-aucune disposition réglementaire.

Le décret du 25 mars 1924 portant règlement d'administration publique pour l'application de la loi du $1^{\text {er }}$ août 1905 , en ce qui concerne le lait et les produits dérivés du lait, n'avait pu que s'inspirer de ces principes ; il ne faisait pas obstacle, notamment, à ce que des fromages fussent fabriqués avec des matières grasses étrangères au lait, à condition que la dénomination du produit fut accompagnée d'une mention révélatrice.

Le projet de loi de M. Fernand DAvid, comme l'indiquait l'exposé des motifs, a eu pour but d'éliminer du marché ces produits laitiers dont la matière grasse n'était pas constituée par de la pure crème de lait, et cela, en s'appuyant sur des considérations économiques; le consommateur, pensait-on, dans une période de pléthore laitière n'avait nul intérêt à faire appel à des "ersatz ", et le produeteur, lui, avait le plus grand intérêt à ce que ceux-ci fussent exclus.

Voilà pourquoi les dispositions de l'article premier du nouveau texte interdisent de fabriquer et de vendre, sous quelque dénomination que ce soit, des produits laitiers, ayant l'apparence de produits définis (crèmes, fromages, etc.), mais ne s'adaptant pas, par leur composition, aux définitions correspondantes.

La Commission de l'Agriculture de la Chambre, dont le rapporteur était M. LAUvRAY, non seulement approuva le projet de loi qui lui fut soumis, mais encore en renforça les dispositions :

Tout d'abord, elle inséra dans le texte du gouvernement, sous forme d'article 2, une clause interdisant l'emploi du mot « beurre ", dans la publicité et dans les annonces relatives au commerce des matières grasses comestibles autres que le beurre. Il ne sera plus permis, désormais, de vendre une de ees substances sous une étiquette portant la mention : "meilleur que le beurre", par exemple. 
Ensuite, la Commission ajouta au projet un article 3 ainsi conçu : " Est interdite l'importation de produits laitiers provenant du pays " où la fabrication de la margarine n'est pas soumise au contrôle "permanent d'un service d'inspection et où on n'incorpore pas à " celle-ei un produit révélateur susceptible de la faire reconnaître "facilement dans un mélange même à des doses très faibles. ")

Les produits laitiers importés devront être contenus dans des emballages sur lesquels sera inscrit, en earactères très apparents, le nom du pays d'origine.

Si cet article devait être appliqué très rigoureusement, des pays exportateurs de beurres et de fromages, qui ne posséderaient pas une législation identique à la nôtre en ce qui concerne la fabrication de la margarine (addition d'une substance révélatrice, susceptible de faire découvrir aisément la fraude, en cas de mélange avec du beurre ou de la crème), ne pourraient plus expédier ces produits chez nous, même s'ils avaient, sur leur propre territoire, un contrôle sévère et des garanties équivalentes aux nôtres contre la falsification.

Mais M. Queville, Ministre de l'Agriculture, au moment où le projet de loi, adopté par la Chambre dans la séance du 30 mars 1932, est venu en discussion devant le Sénat (séance du 25 juin 1934), a formulé les déclarations suivantes au sujet dudit article $3:$ :Faudra" $\mathrm{t}$-il que les beurres hollandais et les fromages hollandais ne puis" sent plus venir en France ?... Je serai certainement d'accord avec " la Commission de l'Agriculture et M. Fernand DAvid en disant que "si la Hollande nous donne des garanties équivalentes à celles que le projet prévoit, nous envisagerons des mesures de conciliation.

"Au reste, ce que nous lui demandons, c'est de faire un effort "pour que, ses produits étant reçus en France, elle reçoive à son " tour et avec la même bienveillance, des produits agricoles français. "Sur la base d'une équitable réciprocité de cette nature, nous ne " manquerons pas de faire preuve, même dans l'application de ce qui " serait la loi de demain, de l'esprit de conciliation indispensable."

L'ensemble des nouveaux textes a été adopté par le Sénat, malgré les réserves exprimées au nom de la Commission du Commeree de cette assemblée par M. Paul LAFFonT, et après des discours favorables de M. Donon, l'éminent rapporteur de la Commission de l'Agriculture, de M. le Sénateur DAMEcodr, et du Ministre lui-même.

Cette loi du 29 juin 1934, dépassant sensiblement le cadre des prévisions du législateur de 1905 , est à rapprocher de la série de celles qui, en réglementant étroitement, ou même en prohibant la vente de certains produits d'imitation, ont considérablement renforcé, depuis quelques années, l'arsenal de la répression des fraudes. 